\title{
Reducing Perinatal Deaths: A Distant Dream But on the Right Path!
}

\author{
SRINIVAS MURKI ${ }^{1 *}$ and SANJEev Upadhyaya ${ }^{2}$ \\ ${ }^{1}$ Chief Neonatologist, Paramitha Children Hospital, and ${ }^{2}$ Health Specialist \\ UNICEF Office for Andhra Pradesh, Telangana \& Karnataka; Hyderabad, Telangana, India. \\ *srinivasmurki2001@gmail.com
}

\section{"In God we trust, all others bring data!"}

$\Gamma$ he phrase emphasizes the importance of 'data' to understand a problem, identify the causes, implement preventive and therapeutic activities and specially to plan health programs that concern public health issues. Data collection is an ongoing systematic process of gathering, analyzing and interpreting various types of information from relevant sources. Quality assurance and quality control are two important components of any data. Quality assurance starts before the data collection and quality control occurs during and after data collection. The study by Kumar, et al. [1] published in the current issue of Indian Pediatrics, the authors evaluated the quality assurance aspect of a perinatal mortality audit. They evaluated the available resources including manpower (public and private hospital staff), protocols (registries, case-sheets, certification), training and reporting (reports generated) required for audit of perinatal mortality. The study shows major gaps in the documentation and reporting of peri-natal deaths. The reasons for lack of quality assurance on perinatal mortality audit from the current study include recent introduction of facility based CDR (child death review) or community based CDR of neonatal deaths and absence of any policy on still birth review till recent times [2]. Inclusion of many private hospitals in the audit also seems to be a major reason for identifying wider systemic gaps. The present study as well as other Indian studies have highlighted the poor engagement of private sector in maternal and infant death reviews, although the private sector contributes to $43 \%$ of deliveries in urban areas and $22 \%$ deliveries (NHFS 4) in rural areas [3].

Perinatal mortality (PMR) includes still births and early neonatal deaths. As the cause of mortality for these components are closely related to maternal antenatal and intrapartum care, PMR is considered one of the important health indicators of quality of care around delivery. The current PMR of the country is 36 per 1000 pregnancies (NFHS -4). Although reduction of PMR is not addressed directly, the health programs in the country are largely directed to reduce still births and neonatal deaths. India newborn action plan (INAP) targets to reduce still birth rate (SBR) and neonatal mortality rate (NMR) to a single digit by 2030 [4]. Annually approximately 6 lac still births occur in the country and of these nearly $45 \%$ occur during delivery. As per the estimates published in Lancet in 2011, the SBR in India is 22 per 1000 total births [5]. The portal of the Health management information system (HMIS) published by the Ministry of health and family welfare, Government of India (GOI), reported 3,03,857 stillbirths for the period 2015-16, which seems to be a gross underestimate [6]. A sentinel still-birth surveillance program launched by GOI in June 2016, not only provides an opportunity to count stillbirths but also helps to review the circumstances, risk factors and leading determinants resulting in a still born baby [6]. Data from this surveillance will be of immense use to meet the India newborn action plan (INAP) target of single digit SBR by 2030 . However, the known causes of stillbirths are largely addressed in the existing health and other social sector programs directly or indirectly. Janani suraksha yojana (JSY) program ensures registration of all pregnant mothers, encourages antenatal visits, and institutional delivery with cash incentives. The success of this program is visible in most states as institutional deliveries in public sectors hospitals improved signifi-cantly over the last decade. Also, NFHS-4 revealed that the proportion of institutional deliveries (public or private) almost doubled from 39\% in 2005-06 to $79 \%$ in 2015-16 [7]. The Dakshata program, a skill oriented, evidence-based, woman- and baby-friendly training ensures essential interventions that need to be in place to reduce reproductive, maternal, newborn and child mortality and morbidity and promote reproductive health. In addition to JSY and the Dakshata program, the Labour room and quality improvement initiative (LaQshya) and the Pradhan mantri surakshit matritva abhiyan (PMSMA) would contribute significantly to reduce SBR [8].

Neonatal mortality and early neonatal mortality are adequately addressed in the current health programs of 
the country. Facility based death review (FBDR) and Community based death review (CBDR), under the National health mission are important policy decisions to account for neonatal deaths. Appropriate implementation and feedback of these would help in improving community awareness, reduce the gap between facility and the pregnant mother, improve services to cater to the care of newborn and also integrate other social services such as sanitation, nutrition and availability of potable drinking water. Navjaat shishu suraksha karyakram (NSSK), a capacity building program implemented in collaboration with Indian academy of pediatrics (IAP), backed with a scheme like Janani shishu suraksha karyakram (JSSK) and state of the art infrastructure in the form of Special newborn care units (SNCUs), Newborn stabilization units (NBSUs) and the Newborn care corners (NBCCs) across the country have contributed significantly in reducing the newborn deaths due to asphyxia, prematurity and sepsis [8]. There is still a great need to improve newborn care further across different tiers of health facilities to achieve the INAP targets by 2030.

In conclusion, the present paper brings out glaring deficiencies in documentation, record keeping and reporting of perinatal deaths as on 2015, but a significant change is visible in the last five years with the inclusion of child death review in NHM, and also the start of a sentinel surveillance of stillbirths. We sincerely hope that the upcoming National digital health mission (NDHM) will ensure that these deficiencies wouldn't exist in coming years [8]. Since neonatal mortality rate is an INAP indicator, and is closely monitored under current national programme, there are instances to (mis)classify early neonatal deaths as stillbirths so as to keep the neonatal mortality low. This makes a strong case to begin monitoring the perinatal mortality rate across the country and expand the scope of CDR to include stillbirths. Nodal officers for CDR should be made more responsible for perinatal death reviews at state, district and sub-district Level.

Funding: None; Competing interests: None stated

\section{REFERENCES}

1. Kumar HNH, Baliga S, Kushtagi P, et al. Documentation and reporting of derinatal Deaths in two districts of Karnataka, India: A situational analysis. Indian Pediatr; 2020;57:1006-09.

2. Child Health Division: Ministry of Health and Family Welfare, Government of India. Child Death Review. Operational Guidelines. August 2014. Available from http://pbhealth.gov.in/Operational\%20Guide\%20of\%20 Child\%20Death\%20Review.pdf. Accessed July 31, 2020.

3. Dhirar N, Dudeja S, Khandekar J, Bachani D. Childhood Morbidity and Mortality in India - Analysis of National Family Health Survey 4 (NFHS-4) Findings. Indian Pediatr. 2018;55:335-38.

4. India Newborn Action Plan (INAP). National Health Mission. September 2014. MOHFW, GOI Publication. Available from: https://nhm.gov.in/images/pdf/programmes/ inap-final.pdf. Accessed July 31, 2020.

5. Lawn JE, Blencowe H, Pattinson R, et al. Stillbirths: Where? When? Why? How to make the data count?. Lancet. 2011; 377:1448-63.

6. Child Health Division: Ministry of Health and Family Welfare, Government of India. Operational guidelines for establishing Sentinel Stillbirth Surveillance System. June 2016. Available from: https://www.nhm.gov.in/images/pdf/ programmes/RBSK/Operational_Guidelines/Operational_ Guidelines_for_establishing_Sentinel_Stillbirth_Surveillance_ System.pdf. Accessed on July 31, 2020.

7. Gupta SK, Pal DK, Tiwari R, et al. Impact of Janani Suraksha Yojana on institutional delivery rate and maternal morbidity and mortality: an observational study in India. J Health Popul Nutr. 2012;30:464-71.

8. Facility Based Newborn Care (FBNC) Operational Guide. National Health Mission. MOHFW, GOI Publication. Available from:https://nhm.gov.in. Accessed on August 10, 2020. 